\title{
Parameter Selection Guidelines for a Parabolic Sliding Mode Filter Based on Frequency and Time Domain Characteristics
}

\author{
Shanhai Jin, Ryo Kikuuwe, and Motoji Yamamoto \\ Department of Mechanical Engineering, Kyushu University, Motooka 744, Nishi-ku, Fukuoka 819-0395, Japan \\ Correspondence should be addressed to Shanhai Jin, jin@ctrl.mech.kyushu-u.ac.jp
}

Received 1 August 2012; Revised 29 October 2012; Accepted 4 November 2012

Academic Editor: Mohamed Zribi

Copyright () 2012 Shanhai Jin et al. This is an open access article distributed under the Creative Commons Attribution License, which permits unrestricted use, distribution, and reproduction in any medium, provided the original work is properly cited.

This paper presents the results of quantitative performance evaluation of an authors' new parabolic sliding mode filter, which is for removing noise from signals in robotics and mechatronics applications, based on the frequency and time domain characteristics. Based on the evaluation results, the paper presents selection guidelines of two parameters of the filter. The evaluation results show that, in the frequency domain, the noise removing capability of the filter is almost the same as that of the second-order Butterworth low-pass filter (2-LPF), but its phase lag is smaller (maximum 150 degree) than that of 2-LPF (maximum 180 degree). Moreover, the filter produces smaller phase lag than a conventional parabolic sliding mode filter with appropriate selection of the parameters. In the time domain, the filter produces smaller overshoot than 2-LPF and the conventional one, while maintaining short transient time, by using an appropriately selected parameter. The presented parameter selection guidelines state that the values of the parameters should be chosen according to some estimated characteristics of the input and some desired characteristics of the output. The effectiveness of the filter and the presented guidelines is validated through numerical examples and their application to a closed-loop, force control of a robot manipulator.

\section{Introduction}

In many robotics and mechatronics applications, filters are required for removing noise component from measured signals. Linear filters are widely used for removing noise due to their simplicity. They, however, proportionally transfer any noise component into the output. Besides that, strong attenuation of noise usually results in a large phase lag, which may lead to instability of closed-loop systems. Such drawbacks of linear filters may cause undesirable problems in many applications.

Some nonlinear filters have been used for circumventing drawbacks of linear filters. For example, the median filter [1] is known to be useful in removing impulsive noise but it is computationally expensive, as pointed out in [2]. As another example, Janabi-Sharifi et al. [3] proposed an adaptive windowing filter that is designed to optimize the trade-off between filtering effectiveness and filtering time delay. The computational cost of their filter, however, depends on the sampling interval and the input frequency. Stochastic filters, such as Kalman filter [4-7], can be useful in some applications. They, however, require a dynamics model of the source of the signal, which is not always obtained, and their performance depends on the accuracy of the model.

Some researchers study sliding mode observers as filters for removing noise from signals (e.g., [8-10]). In sliding mode observers, the observer state (typically consisting of the output and its derivative) is forced to reach a predetermined surface, which is called sliding surface, in the state space. Once the observer state reaches the sliding surface, the state is constrained to the sliding surface and it slides along the surface to a desired state. Thus, the observer state becomes insensitive to noise. In the last decade, much attention has been paid to the study of sliding mode observers based on the super-twisting algorithm (e.g., [11-13]). These observers theoretically realize finite time convergence in continuous-time analysis. However, the accuracy of convergence in discrete-time implementation, typically with 
Euler discretization (e.g., [14-16]), depends on the sampling interval, as pointed out in [11]. In addition, they are prone to overshoot during the convergence.

The sliding mode filter employing a parabolic-shaped sliding surface, which will be explained in Section 2, has also been studied. This filter was independently proposed by Han and Wang [17] and Emaru and Tsuchiya [18, 19] (see also [20] for some discussion). After that, some evaluation results of their filter [21-23] and its applications [24-29] were reported. The main advantage of their filter is that it realizes finite time convergence of the output to the input value when a constant input is provided. Their filter, however, is prone to overshoot because the state is attracted to the sliding surface only from one side.

In a previous paper [30], the authors proposed a new parabolic sliding mode filter, which is an extension of the filter [17-19]. After that, in another previous paper [31], the effectiveness of this filter in improving velocity feedback in position control of a device was demonstrated by the authors. In $[30,31]$, however, the performance of this filter is not quantitatively evaluated, and any guideline for selecting the parameters is not provided.

In this paper, the performance of the authors' new parabolic sliding mode filter is quantitatively evaluated based on its frequency and time domain characteristics. Based on these evaluation results, selecting guidelines for two parameters are presented. In addition, numerical examples and experimental results are presented.

The rest of this paper is organized as follows. Section 2 gives a brief overview of parabolic sliding mode filters. Sections 3 and 4 evaluate the performance of the authors' new filter based on the frequency and time domain characteristics, respectively. Section 5 gives parameter selection guidelines based on the performance evaluation results. Sections 6 and 7 validate the effectiveness of the new filter and the presented guidelines through numerical examples and experiments. Section 8 provides concluding remarks.

\section{Overview of Parabolic Sliding Mode Filters}

In a previous paper [30], the authors proposed a sliding mode filter of which continuous-time representation is given as follows:

$$
\begin{aligned}
& \dot{x}_{1}=x_{2}, \\
& \dot{x}_{2}=-F \operatorname{gsgn}\left(\operatorname{gsgn}\left(-\alpha, x_{2},-1\right),\right. \\
& \left.\qquad \sigma\left(F, x_{1}, x_{2}, u\right), \operatorname{gsgn}\left(1, x_{2}, \alpha\right)\right), \\
& y=x_{1},
\end{aligned}
$$

where

$$
\sigma\left(F, x_{1}, x_{2}, u\right) \triangleq 2 F\left(x_{1}-u\right)+\left|x_{2}\right| x_{2}
$$

Here, $u \in \mathbb{R}$ is the input, $y \in \mathbb{R}$ is the output, $x_{1} \in \mathbb{R}$ and $x_{2} \in \mathbb{R}$ are the states of the filter, and $F>0$ and $\alpha>1$ are parameters to be selected appropriately. In addition, gsgn

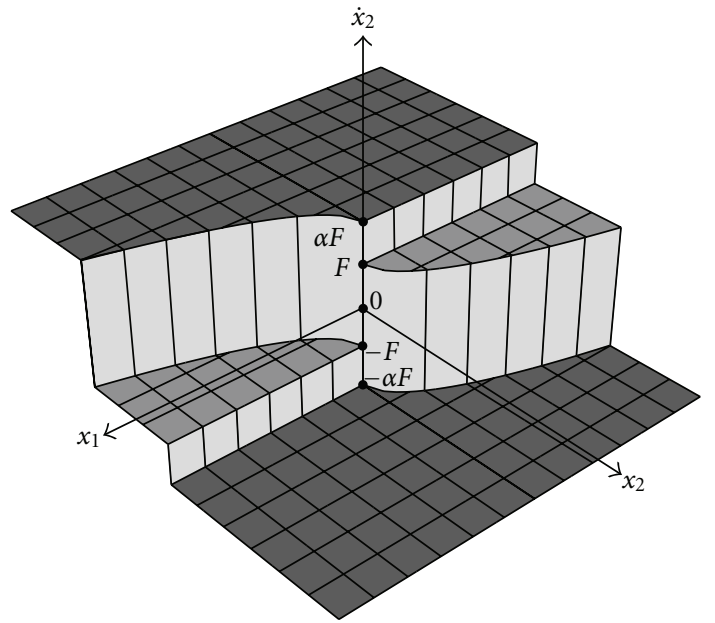

FIgURE 1: Relation among $x_{1}, x_{2}, \dot{x}_{2}$, and $\alpha$.

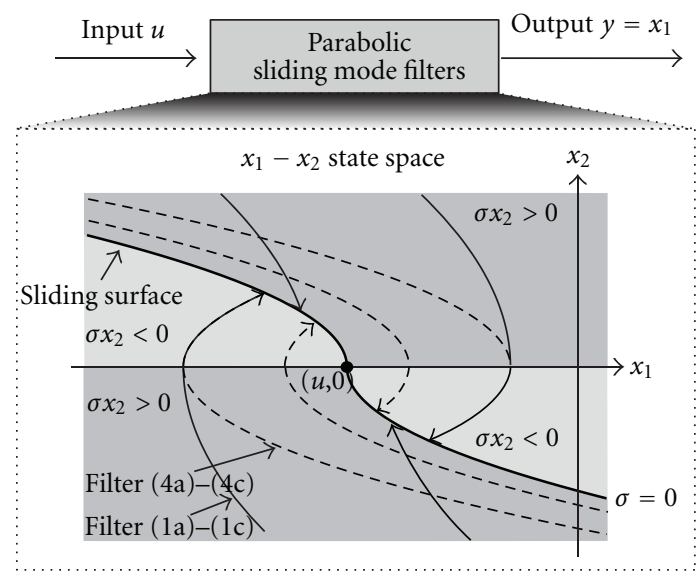

FIGURE 2: The parabolic-shaped sliding surface (thick solid curve, $\sigma=0)$ and trajectories of the state $\left(x_{1}, x_{2}\right)$ for $\sigma \neq 0$ in filter (1a)(1c) (thin solid curve) and filter (4a)-(4c) (thin dotted curve).

is the set-valued, generalized signum function defined as follows:

$$
\operatorname{gsgn}(A, z, B) \triangleq \begin{cases}B & \text { if } z>0 \\ {[\min (A \cup B), \max (A \cup B)]} & \text { if } z=0 \\ A & \text { if } z<0\end{cases}
$$

where the arguments $A \subset \mathbb{R}$ and $B \subset \mathbb{R}$ are arbitrary closed intervals (which can be scalars as a special case) and the argument $z \in \mathbb{R}$ is a scalar. It should be noted that, when $z=0$, gsgn returns the union of $A$ and $B$ and all values in between. Figure 1 shows the relation among $x_{1}, x_{2}, \dot{x}_{2}$, and $\alpha$ in filter (1a)-(1c). One can see that $\dot{x}_{2}$ takes the value of either $-\alpha F$ or $\alpha F$ when $\sigma x_{2}>0$, as shown by the horizontal surfaces. In addition, when $\sigma x_{2}=0, \dot{x}_{2}$ takes a value between the values of $\dot{x}_{2}$ in adjoining regions, as shown by the vertical surfaces. 


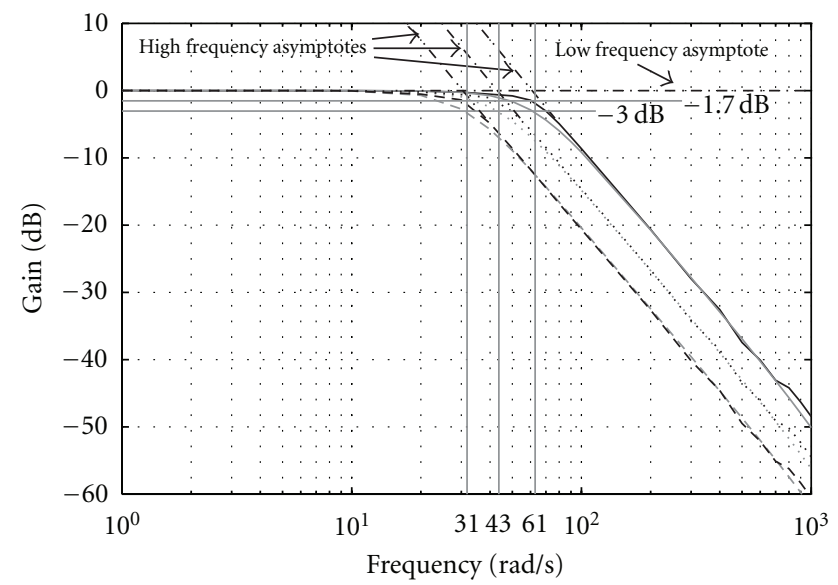

(a) Gain plot

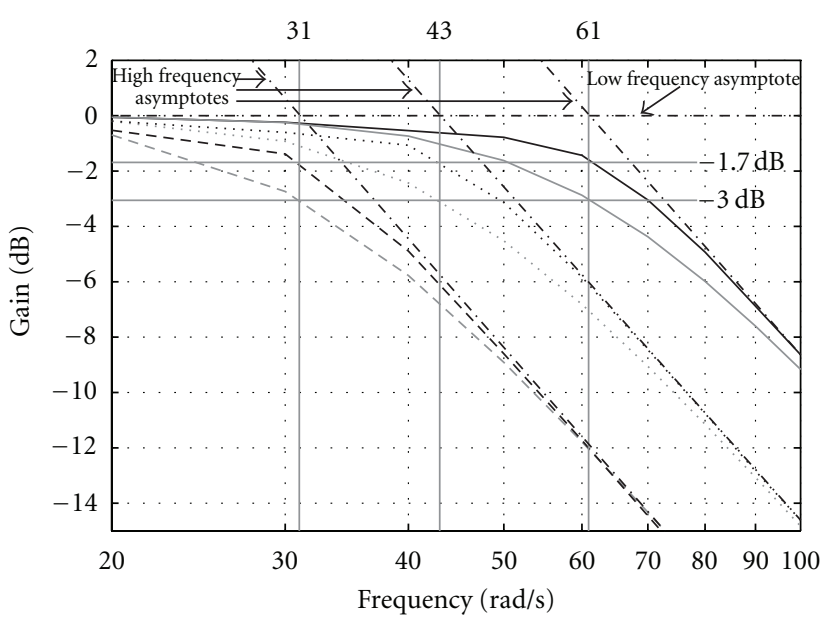

(b) Enlarged view of (a)

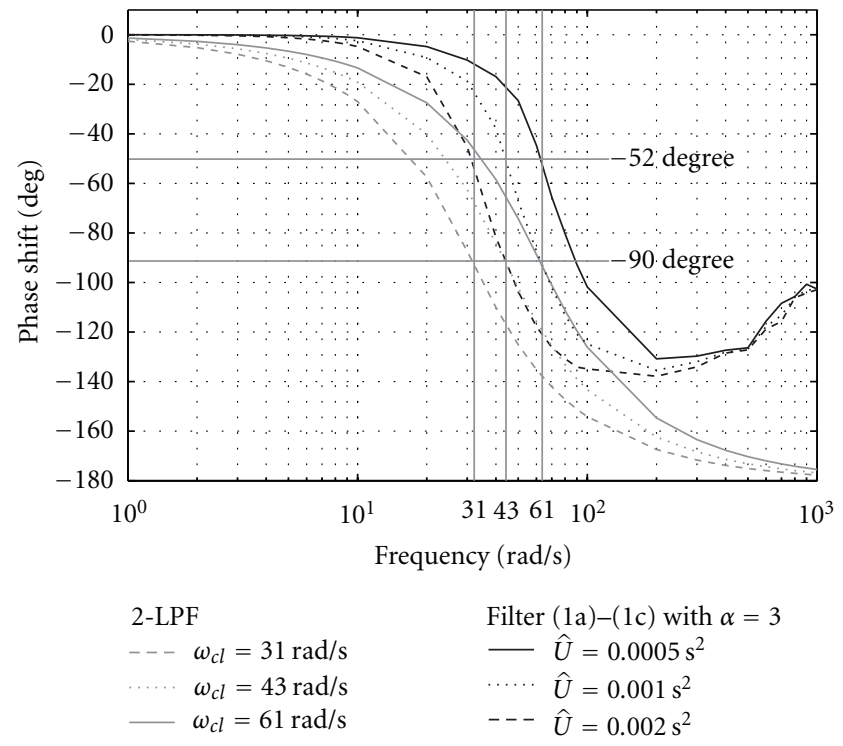

(c) Phase plot

FIGURE 3: Bode plots for different values of $\hat{U}$.

Filter (1a)-(1c) is an extension of a previous parabolic sliding mode filter, which is described as follows:

$$
\begin{aligned}
& \dot{x}_{1}=x_{2}, \\
& \dot{x}_{2}=-F \operatorname{sgn}\left(\sigma\left(F, x_{1}, x_{2}, u\right)\right), \\
& y=x_{1} .
\end{aligned}
$$

Here, $\sigma\left(F, x_{1}, x_{2}, u\right)$ is the same as the one defined in (2), and sgn is the set-valued signum function defined as follows:

$$
\operatorname{sgn}(z) \triangleq \begin{cases}1 & \text { if } z>0 \\ {[-1,1]} & \text { if } z=0 \\ -1 & \text { if } z<0\end{cases}
$$

Note that sgn returns an arbitrary value between 1 and -1 when $z=0$. Filter (4a)-(4c) was independently proposed by Han and Wang [17] and Emaru and Tsuchiya [18, 19]. This filter can be viewed as a special case of the authors' filter (1a)-(1c) with $\alpha=1$ because (3) reduces to (5) when $A=$ $[-1,-1]$ and $B=[1,1]$.

Figure 2 shows the sliding surface and trajectories of the state $\left(x_{1}, x_{2}\right)$ in filter $(1 \mathrm{a})-(1 \mathrm{c})$ and filter $(4 \mathrm{a})-(4 \mathrm{c})$. As is shown by the thick solid curve, filter (1a)-(1c) employs a parabolic-shaped sliding surface, which is designed so that the state $\left(x_{1}, x_{2}\right)$ converges to the state $(u, 0)$ in finite time when $u$ is constant. The difference of filter (1a)-(1c) from filter (4a)-(4c) lies in the regions of $\sigma x_{2}>0$, where $\alpha>1$ takes effect. This difference makes the state of filter (1a)(1c) attracted to the sliding surface from both sides, whereas 


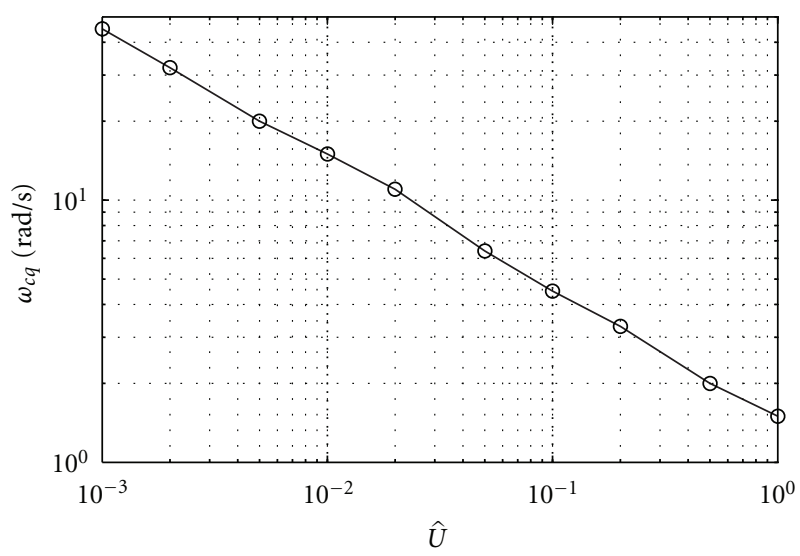

FIGURE 4: Relation between normalized input amplitude $\hat{U}$ and cutoff frequency $\omega_{c q}$ with $\alpha=3$.

the state of filter $(4 a)-(4 c)$ is attracted only from one side, that is, the state of filter $(4 \mathrm{a})-(4 \mathrm{c})$ moves in parallel to the sliding surface in the regions of $\sigma x_{2}>0$. Thus, due to the use of $\alpha>1$, filter (1a)-(1c) is less prone to overshoot than the previous one $(4 a)-(4 c)$.

The continuous-time representation (1a)-(1c) cannot be used directly for numerical computation because it involves the set-valued function gsgn. The authors' previous paper [30] has shown that by using the backward Euler discretization (i.e., replacing $x_{2}$ by $\left.\left(x_{1}(k)-x_{1}(k-1)\right) / T\right)$, (1a)-(1c) can be numerically integrated through the following algorithm:

$$
\begin{aligned}
& x_{2}^{*}(k):=\operatorname{csgn}\left(x_{1}(k-1)-u(k)\right) \\
& \times\left(F T-\sqrt{F^{2} T^{2}+2 F\left|x_{1}(k-1)-u(k)\right|}\right), \\
& x_{2}(k):=x_{2}(k-1) \\
& -\operatorname{gsat}\left(\operatorname{gsat}\left(-\alpha T F, x_{2}(k-1),-T F\right), x_{2}(k-1)\right. \\
& \left.-x_{2}^{*}(k), \operatorname{gsat}\left(T F, x_{2}(k-1), \alpha T F\right)\right) \text {, } \\
& x_{1}(k):=T x_{2}(k)+x_{1}(k-1), \\
& y(k):=x_{1}(k)
\end{aligned}
$$

where $:=$ denotes an assignment of a value, $k$ is the discretetime index, and $T$ is the sampling interval. In addition, gsat is the generalized saturation function [32] defined as follows:

$$
\operatorname{gsat}(a, z, b) \triangleq \begin{cases}b & \text { if } z>b \\ z & \text { if } z \in[a, b] \\ a & \text { if } z<a,\end{cases}
$$

where $a \in \mathbb{R}$ and $b \in \mathbb{R}$ are arbitrary arguments that satisfy $a \leq b$. Besides that, csgn is the conventional signum function defined as follows:

$$
\operatorname{csgn}(z) \triangleq \begin{cases}1 & \text { if } z>0 \\ 0 & \text { if } z=0 \\ -1 & \text { if } z<0\end{cases}
$$

The algorithm (6a), (6b), (6c), and (6d), which is the discrete-time realization of filter (1a)-(1c), does not produce chattering, which has been one of major problems of conventional implementation of sliding mode techniques. This is owing to the use of the backward Euler discretization, which has been recognized to be useful to realize exact reaching to sliding modes as reported in [32-34].

It should be noted that the algorithm (6a), (6b), (6c), and (6d) can also provide $x_{2}(k)$ as an estimated differential value of the input $u(k)$. However, this paper only considers the output $x_{1}(k)$ because $x_{2}(k)$ is the result of simple numerical differentiation of $x_{1}(k)$.

\section{Frequency Domain Analysis}

This section presents the Bode plot of the nonlinear filter (1a)-(1c) by using the describing function method [35, Chapter 7]. Specifically, Sections 3.1, 3.2, and 3.3 investigate Bode plots of filter (1a)-(1c) for different values of $\hat{U}, \alpha$, and sampling interval $T$, respectively. Moreover, Sections 3.1 and 3.2 compare the frequency response of filter (1a)-(1c) to those of the second-order Butterworth low-pass filter (2LPF) and filter (4a)-(4c), respectively. In these two sections, all the three filters are implemented at sampling interval $T=0.001 \mathrm{~s}$ in discrete time.

In the upcoming analysis, the following sinusoidal input signals are used:

$$
u=U \sin (\omega t)
$$

where $U>0$. For simplicity, filter (1a)-(1c) and the input $u$ in (9) are, respectively, normalized as

$$
\begin{aligned}
& \dot{\hat{x}}_{1}=\hat{x}_{2}, \\
& \dot{\hat{x}}_{2}=-\operatorname{gsgn}\left(\operatorname{gsgn}\left(-\alpha, \hat{x}_{2},-1\right),\right. \\
& \left.\qquad \sigma\left(1, \hat{x}_{1}, \hat{x}_{2}, \hat{u}\right), \operatorname{gsgn}\left(1, \hat{x}_{2}, \alpha\right)\right), \\
& \hat{y}=\hat{x}_{1}
\end{aligned}
$$

and

$$
\widehat{u} \triangleq \hat{U} \sin (\omega t)
$$

where

$$
\hat{x}_{1} \triangleq \frac{x_{1}}{F}, \quad \hat{x}_{2} \triangleq \frac{x_{2}}{F}, \quad \hat{u} \triangleq \frac{u}{F}, \quad \hat{U} \triangleq \frac{U}{F} .
$$

This normalization allows us to exclude the influence of $F$ out of consideration without loss of generality. Note that the units of the normalized input $\hat{u}$ and output $\hat{y}$ are $s^{2}$. 


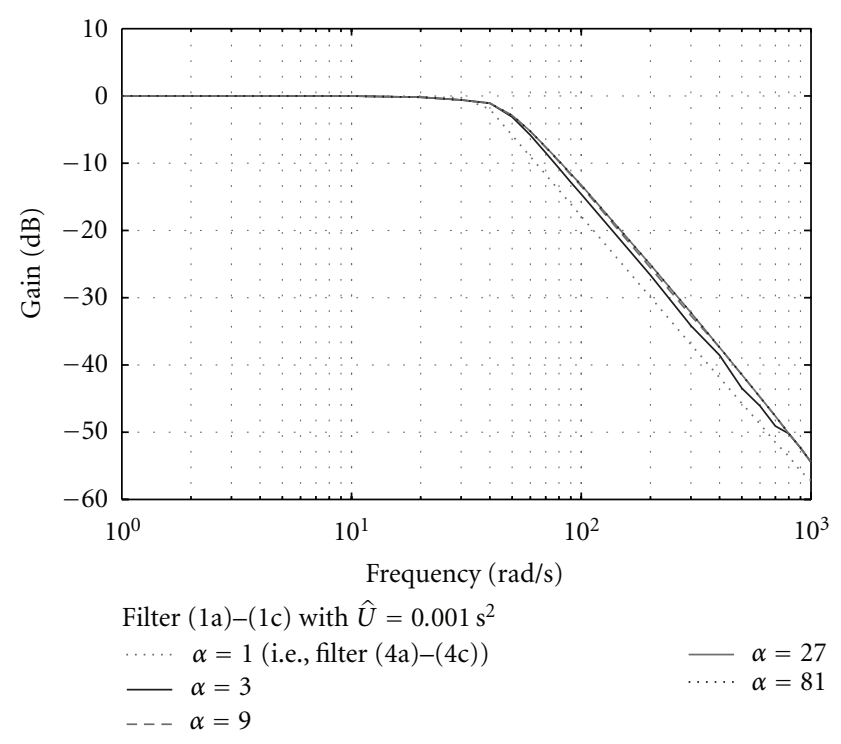

(a) Gain plot for different values of $\alpha$

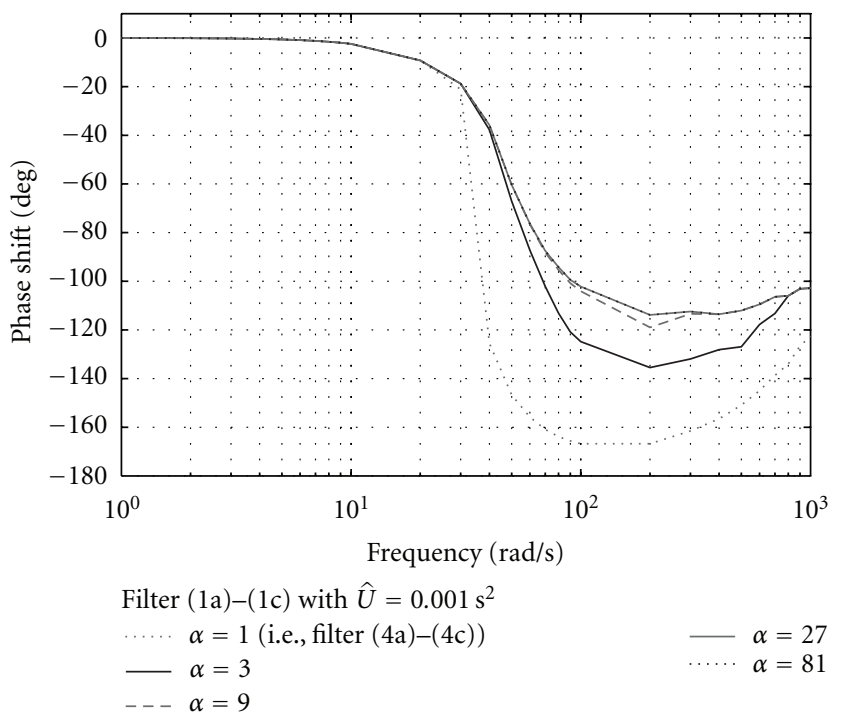

(b) Phase plot for different values of $\alpha$

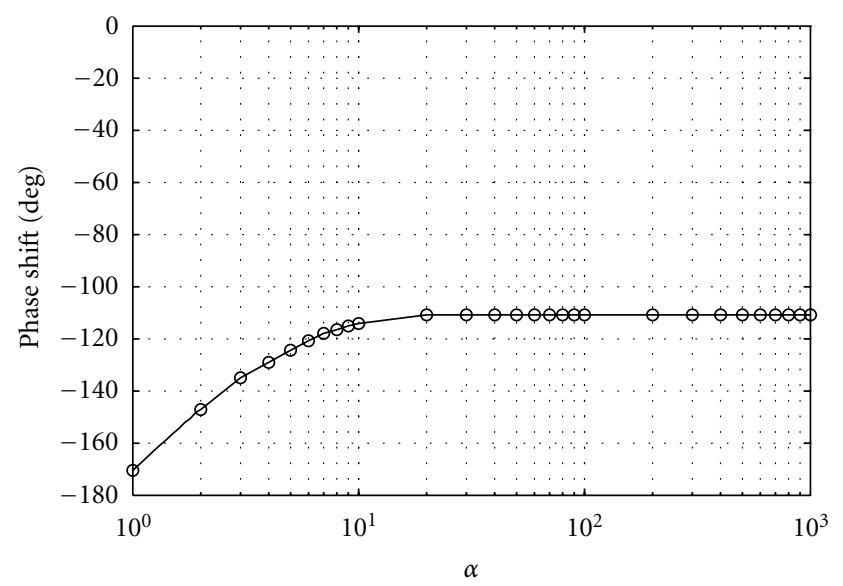

(c) Relation between $\alpha$ and phase shift at $\omega_{c q}=100 \mathrm{rad} / \mathrm{s}$ with $\hat{U}=$ $0.001 \mathrm{~s}^{2}$

FIGURE 5: Influence of $\alpha$ on the gain and the phase shift. In each figure, the result of filter (1a)-(1c) with $\alpha=1$ refers to that of filter (4a)-(4c).

3.1. Bode Plots for Different Values of $\hat{U}$. Figure 3 shows the Bode plots of filter (1a)-(1c) for different values of $\hat{U}$. In filter (1a)-(1c), the frequency response depends on the input amplitude $\hat{U}$ because of the nonlinearity. Specifically, both the gain and the phase plots shift to the left by maintaining the same shape as $\hat{U}$ increases. For comparison, Bode plots of some 2-LPFs are also included in Figure 3. Here, the cutoff frequencies of the 2-LPFs (hereafter, denoted by $\omega_{c l}$ ) are chosen so that their gain plots are as close as possible to those of filter (1a)-(1c). It is shown that, in the gain plot of filter (1a)-(1c), the slopes of the high frequency asymptotes are approximately $-40 \mathrm{~dB} /$ decade, which are the same as that of 2-LPF. In the rest of this paper, for convenience, the term "cut-off frequency" is also used to refer the frequency at which the two asymptotes of filter (1a)-(1c)'s gain plot intersect to each other, and it is denoted by $\omega_{c q}$.

Figures 3(a) and 3(b) show that the gain of filter (1a)(1c) is around $-1.7 \mathrm{~dB}$ at the cut-off frequency $\omega_{c q}$, while that of $2-\mathrm{LPF}$ is around $-3 \mathrm{~dB}$ at the cut-off frequency $\omega_{c l}$. It is known that Butterworth low-pass filters possess the flattest gain plot at cutoff frequencies $\omega_{c l}$ among all linear filters. Thus, it can be said that filter (1a)-(1c) has better gain characteristics than all linear filters. Figure 3(c) shows that the phase shifts of filter (1a)-(1c) and 2-LPF are around -52 degree and around -90 degree, respectively, at their respective cut-off frequencies. In addition, the maximum phase shift of filter (1a)-(1c) is around - 135 degree, whereas that of 2-LPF is around -180 degree. Thus, it also can be concluded that filter (1a)-(1c) produces smaller phase lag than 2-LPF.

Figure 4 shows the relation between $\hat{U}$ and the cut-off frequency $\omega_{c q}$ with $\alpha=3$. It is observed that the relation can be approximated as follows:

$$
\omega_{c q}=\sqrt{\frac{2}{\widehat{U}}}
$$




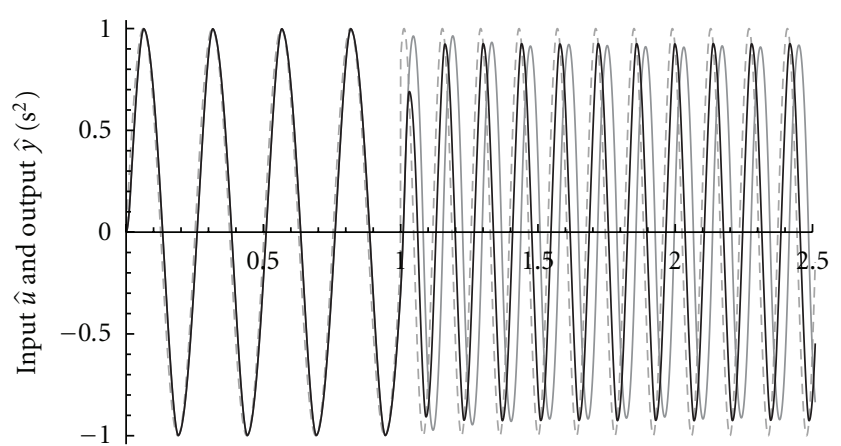

Time (s)

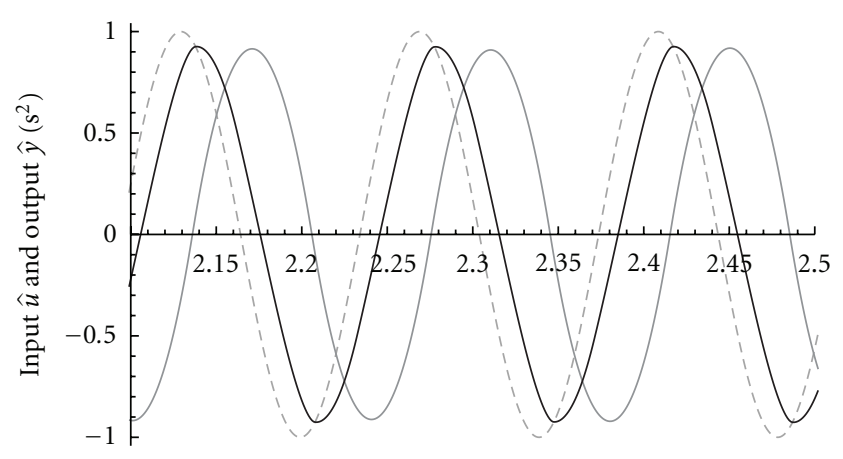

Time (s)

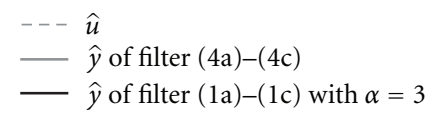

(a) The input and the outputs

(b) Enlarged view of (a)

Figure 6: Comparison between filter (1a)-(1c) and filter $(4 a)-(4 c)$ in the case where the normalized input suddenly changes from $\hat{u}=$ $0.00067 \sin (25 t) \mathrm{s}^{2}$ to $\hat{u}=0.00067 \sin (45 t) \mathrm{s}^{2}$ at $t=1 \mathrm{~s}$.

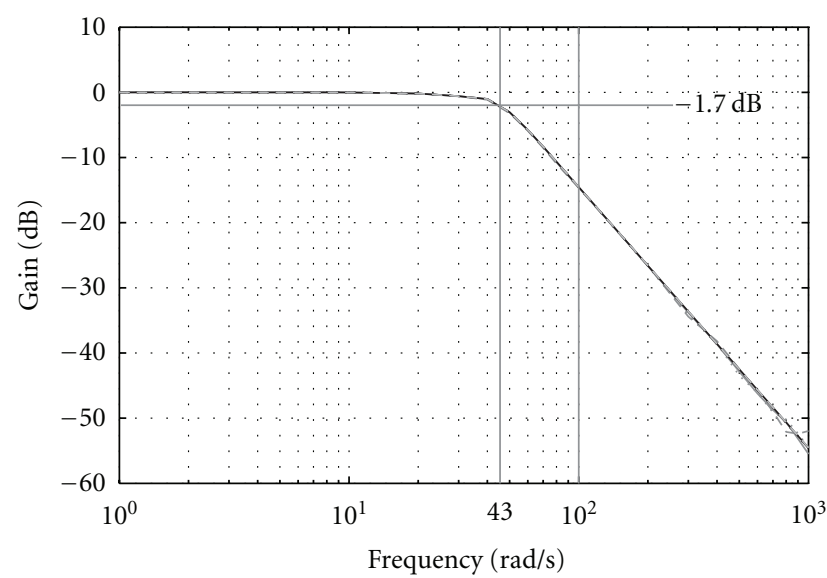

(a) Gain plot

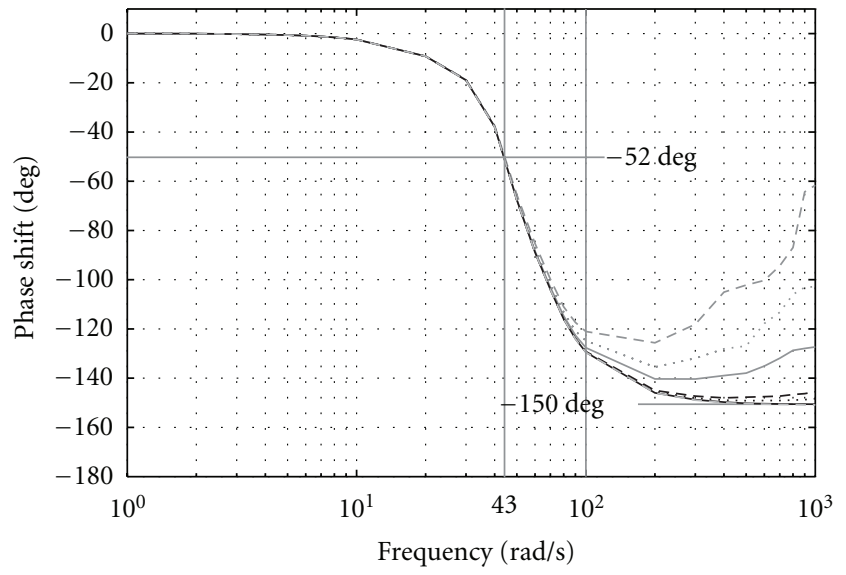

Filter $(1 \mathrm{a})-(1 \mathrm{c})$ with $\hat{U}=0.001 \mathrm{~s}^{2}$
$--T=0.002 \mathrm{~s}$
$\cdots T=0.001 \mathrm{~s}$

(b) Phase plot

FIGURE 7: Bode plots for different values of sampling interval $T$.

In Section 5, the relation (13) will be used in a guideline for selecting the parameter $F$.

3.2. Bode Plots for Different Values of $\alpha$. Figure 5 shows the influence of $\alpha$ on the gain and the phase shift of filter (1a)-(1c). It is observed that, as the value of $\alpha$ increases, the phase lag decreases and asymptotically approaches to a certain value. In particular, one can see that filter $(4 a)-(4 c)$, which is a special case of filter (1a)-(1c) with $\alpha=1$, has similar gain characteristics with that of filter (1a)-(1c), but it produces larger phase lag than filter (1a)-(1c) from the frequency slightly below the cutoff frequency $\omega_{c q}$. Such phase characteristics of the filter (4a)-(4c) may cause instability in closed-loop systems where the frequency range of the input signal is slightly below that of the noise signal.

Figure 6 shows the comparison between filter (1a)-(1c) and filter $(4 a)-(4 c)$ in the case where the normalized input suddenly changes from $\hat{u}=0.00067 \sin (25 t) \mathrm{s}^{2}$ to $\hat{u}=$ $0.00067 \sin (45 t) \mathrm{s}^{2}$ at $t=1 \mathrm{~s}$. Note that both cut-off frequencies of the two filters are $\omega_{c q}=\sqrt{2 / 0.00067} \approx 55 \mathrm{rad} / \mathrm{s}$. It is 


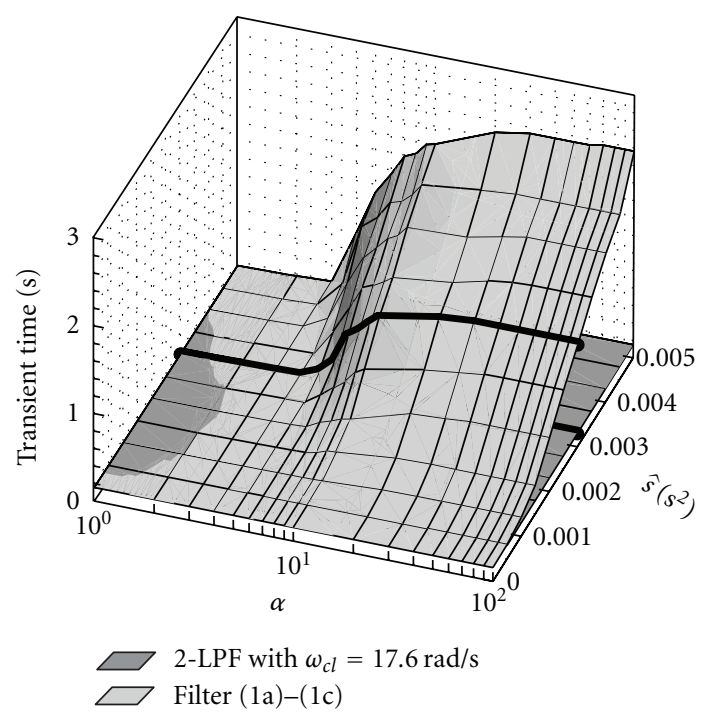

(a) Transient time

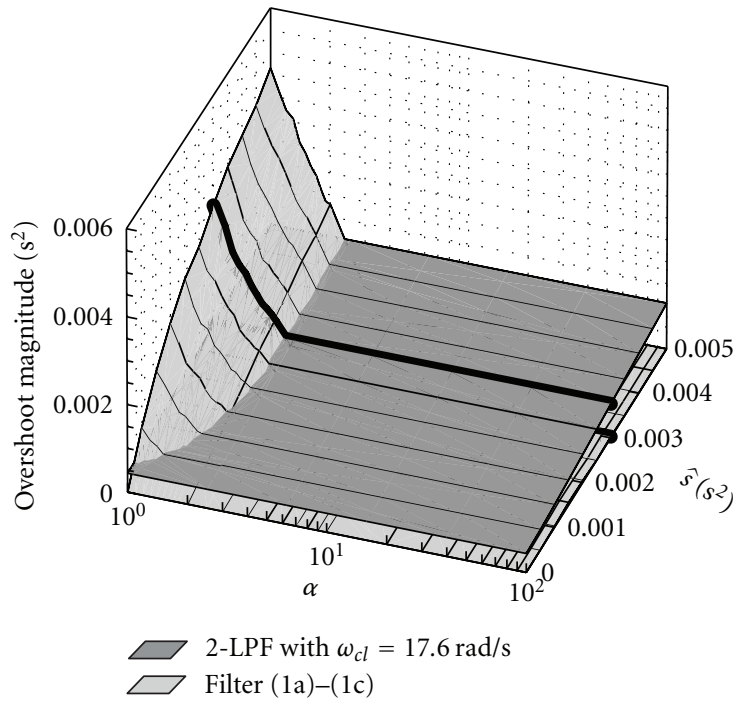

(b) Overshoot magnitude

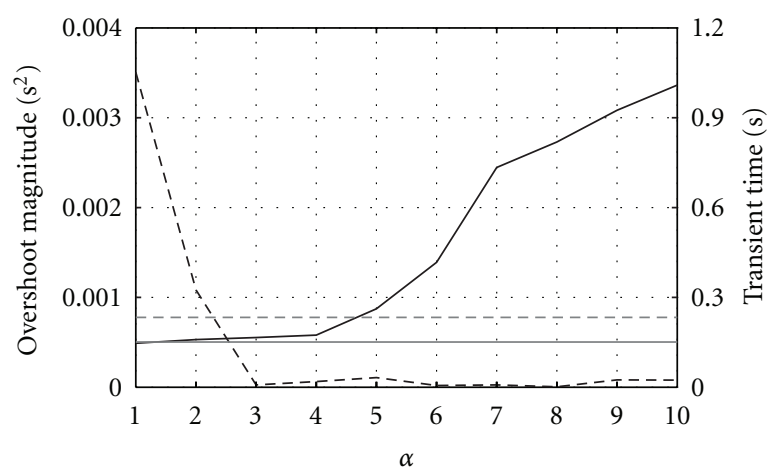

$\begin{array}{ll}\text { 2-LPF with } \omega_{c l}=17.6 \mathrm{rad} / \mathrm{s} & \text { Filter }(1 \mathrm{a})-(1 \mathrm{c}) \\ - \text { Transient time }(\mathrm{s}) & - \text { Transient time }(\mathrm{s}) \\ --- \text { Overshoot magnitude }\left(\mathrm{s}^{2}\right) & --- \text { Overshoot magnitude }\left(\mathrm{s}^{2}\right)\end{array}$

(c) Cross-section view of (a) and (b) at $\hat{s}=0.003 \mathrm{~s}^{2}$

FIGURE 8: Transient time and overshoot magnitude with normalized input $\widehat{u}(t)=0.01+\widehat{s} \varepsilon(t) \mathrm{s}^{2}$, which is a step input corrupted by white Gaussian noise. In each figure, the result of filter (1a)-(1c) with $\alpha=1$ refers to that of filter $(4 a)-(4 c)$.

shown that after the sudden change in the input frequency, filter $(4 a)-(4 c)$ produces larger phase lag than filter (1a)(1c).

3.3. Bode Plots for Different Values of Sampling Interval T. Figure 7 shows the Bode plots of filter (1a)-(1c) for different values of sampling interval $T$. It is shown that the influence of sampling interval $T$ on the gain and the phase shift of filter (1a)-(1c) is insignificant at low frequency range (i.e., frequencies below $\omega=100 \mathrm{rad} / \mathrm{s}$ in the case of $\hat{U}=$ $0.001 \mathrm{~s}^{2}$ ). In particular, with different values of $T$, the gains and the phase shifts at cut-off frequency $\omega_{c q} \approx 43 \mathrm{rad} / \mathrm{s}$ are still around $-1.7 \mathrm{~dB}$ and -52 degree, respectively. It is also shown that at high frequency range (i.e., frequencies above $\omega=100 \mathrm{rad} / \mathrm{s}$ in the case of $\hat{U}=0.001 \mathrm{~s}^{2}$ ), the phase shift decreases and converges to a certain value -150 degree with the increase of $T$. The significant differences of phase shift appeared with long $T$ values are presumably due to the fact that a long $T$ cannot provide enough sampling points to reconstruct a high frequency input signal. Thus, it is advisable to set the sampling interval $T$ as short as a physical system permits to avoid undesirable problems caused by insufficient sampling points, while increasing $\alpha$ to decrease the phase lag as discussed in Section 3.2.

\section{Time Domain Analysis}

This section investigates the transient time and the overshoot magnitude produced by filter (1a)-(1c) according to the following input:

$$
\widehat{u}(t)=0.01+\widehat{s} \varepsilon(t) s^{2},
$$

where $\mathcal{\varepsilon} \sim \mathcal{N}(0,1)$ is the unit white Gaussian noise with zero mean and $\hat{s}$ is a normalized noise-scaling constant. Here, the transient time is defined as the time spent for the output to reach $90 \%$ of the input amplitude with $\hat{s}=0$. 


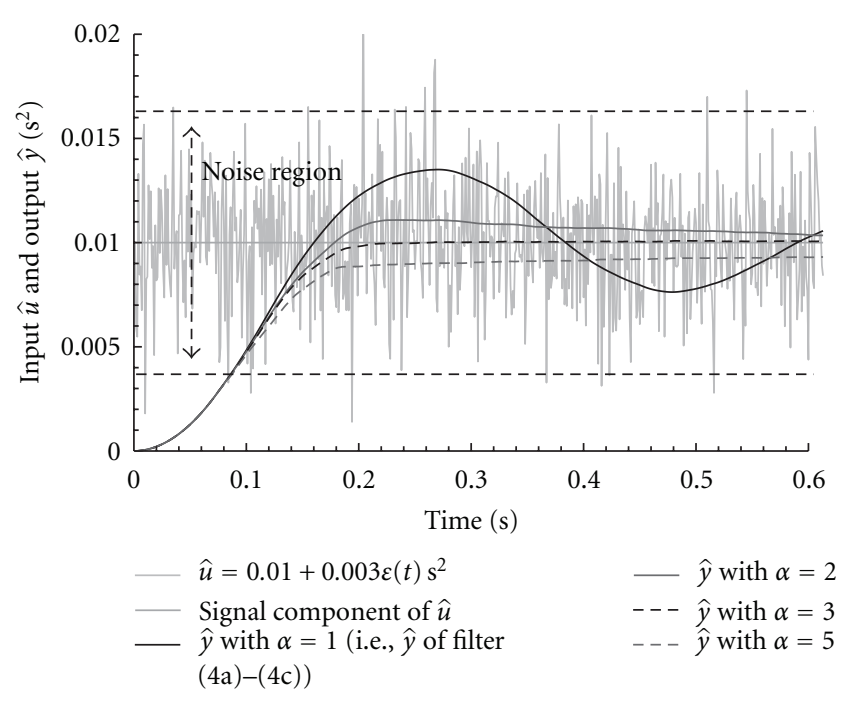

FIGURE 9: Time response of filter (1a)-(1c) with normalized input $\widehat{u}(t)=0.01+0.003 \varepsilon(t) \mathrm{s}^{2}$, which is a step input corrupted by white Gaussian noise. Here, the response of filter (1a)-(1c) with $\alpha=1$ refers to that of filter $(4 a)-(4 c)$.

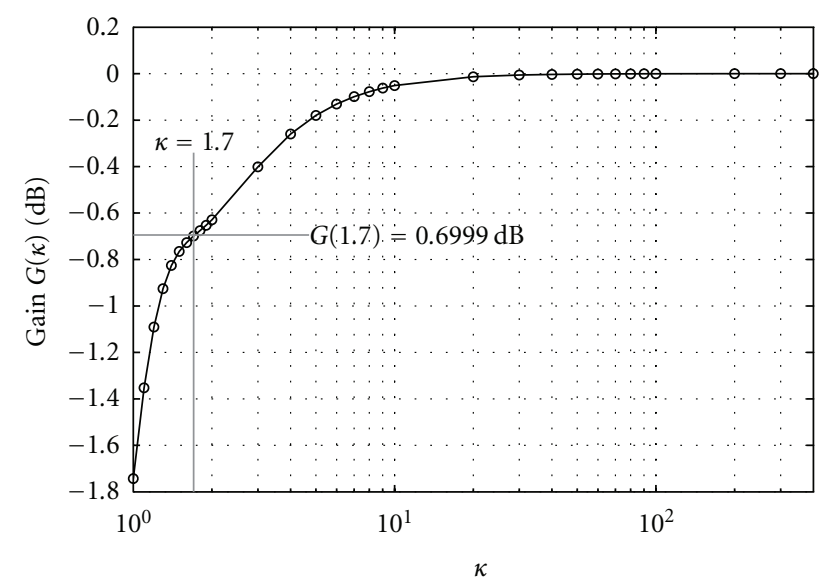

FIGURE 10: Gain $G=\Gamma(\kappa)$ as a function of a parameter $\kappa \geq 1$, with which $F$ is chosen as $F=\kappa \omega_{s}^{2} U_{s} / 2$ and $\alpha$ is set as $\alpha=3(T=0.001 \mathrm{~s})$.

Figure 8 shows the transient time and the overshoot magnitude of filter (1a)-(1c) for different values of $\alpha$ and a 2LPF with the input (14) for different values of $\widehat{s}$. In 2-LPF, the cut-off frequency $\omega_{c l}$ is chosen as $\omega_{c l}=17.6 \mathrm{rad} / \mathrm{s}$ so that the transient time of 2-LPF is as close as possible to that of filter (1a)-(1c) with $\hat{s}=0$. The sampling interval $T=0.001 \mathrm{~s}$ is used for the discrete-time implementation of the two filters.

In Figure 8 , it is clearly shown that the range of optimum values for $\alpha$ is around $3 \leq \alpha \leq 5$. The $\alpha$ values larger than this range result in long transient time and those smaller than this range result in large overshoot magnitude. This tendency becomes stronger as $\hat{s}$ increases. Within this range of $\alpha$, filter (1a)-(1c) produces slightly longer transient time but smaller overshoot magnitude than 2-LPF.
Figure 9 shows some examples of time responses of filter (1a)-(1c) with normalized input $\hat{u}(t)=0.01+0.003 \varepsilon(t) \mathrm{s}^{2}$. It is shown that the influence of $\alpha$ starts when the output $\hat{y}$ enters the region that the input $\hat{u}$ can reach (this region is indicated as a "noise region" in Figure 9). One can see that a larger $\alpha$ leads to a stronger slowing down of $\hat{y}$ and thus leads to a longer transient time and a smaller overshoot magnitude.

\section{Parameter Selection Guidelines}

Based on the discussion in Sections 3 and 4, this section presents selecting guidelines for the two parameters $F$ and $\alpha$ of filter (1a)-(1c). Let us define the following quantities:

$\omega_{s}$ : maximum angular frequency of the signal component in the input,

$U_{s}$ : maximum amplitude of the signal component in the input,

$\omega_{n}$ : minimum angular frequency of the noise component in the input,

$U_{n}$ : maximum amplitude of the noise component in the input,

$U_{n p}$ : maximum permissible amplitude of the noise component in the output, and

$G_{p}$ : minimum permissible gain of the signal component in the output.

Here, we assume $\omega_{s}<\omega_{n}$, that is, the frequency range of the noise component is higher than that of the signal component, and $G_{p}<0 \mathrm{~dB}$. By using these notations, requirements for filter (1a) - (1c) can be described as follows:

(a) the gain $G$ of filter (1a)-(1c) at $\omega_{n}$ with $U_{n}$ should be smaller than $U_{n p} / U_{n}$, and

(b) the gain $G$ of filter (1a)-(1c) at $\omega_{s}$ with $U_{s}$ should be $G_{p}<G<0 \mathrm{~dB}$.

Given these requirements, parameter selection guidelines are now derived. First, Section 3.1 has shown that the gain plot of filter (1a)-(1c) has a high frequency asymptote of which the slope is approximately $-40 \mathrm{~dB} /$ decade, that is, the gain $G$ at a frequency $\omega>\omega_{c q}$ can be approximated as follows:

$$
\begin{aligned}
& 20 \log _{10} G=-40 \log _{10} \frac{\omega}{\omega_{c q}} \\
& \Leftrightarrow G=\frac{\omega_{c q}^{2}}{\omega^{2}} .
\end{aligned}
$$

By using (13) in (15), one can see that the gain at $\omega_{n}$ with $U_{n}$ is $G=2 F /\left(\omega_{n}^{2} U_{n}\right)$. Thus, to achieve $G<U_{n p} / U_{n}, F$ should satisfy $F<\omega_{n}^{2} U_{n p} / 2$.

Next, when the signal with a frequency $\omega_{s}$ and an amplitude $U_{s}$ is provided, the gain $G$ increases as $F$ increases. Figure 10 shows the gain $G=\Gamma(\kappa)$ as a function of a parameter $\kappa \geq 1$, with which $F$ is chosen as $F=\kappa \omega_{s}^{2} U_{s} / 2$. If one needs to obtain a gain larger than $G_{p}$, one should choose 


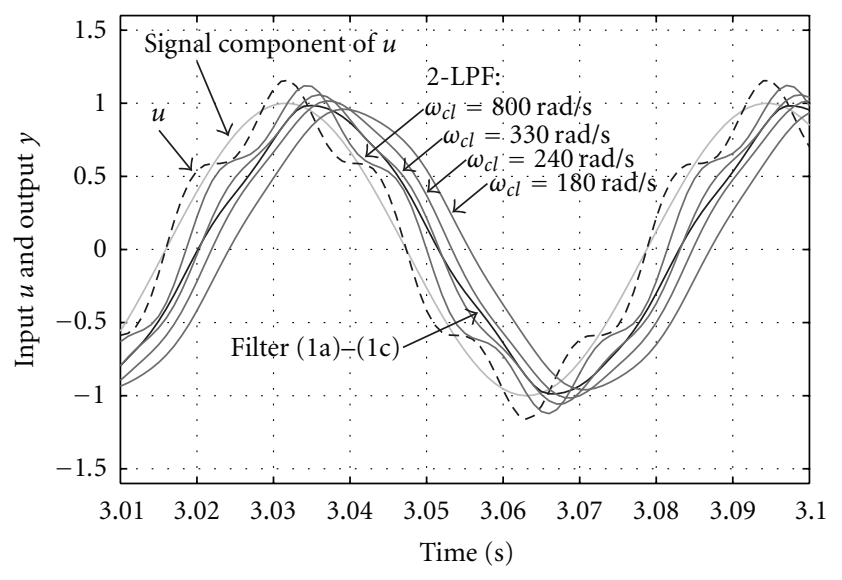

(a) The input and the outputs

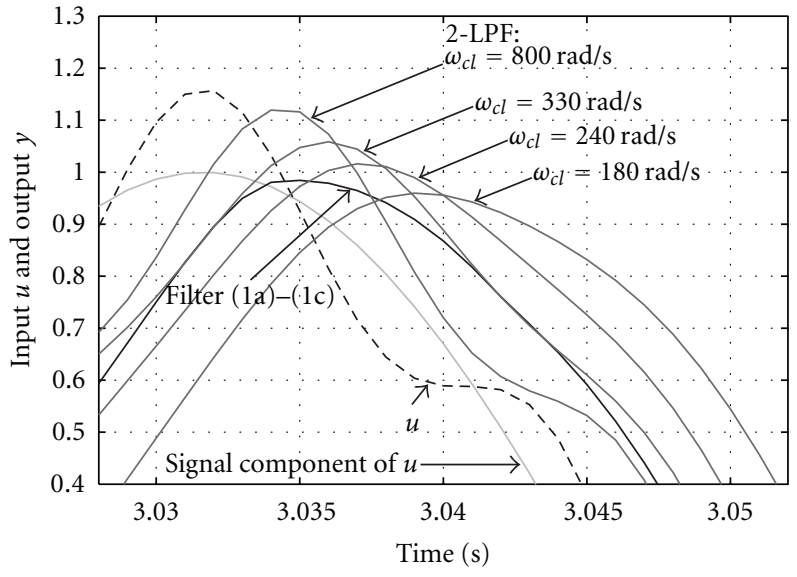

(b) Enlarged view of (a)

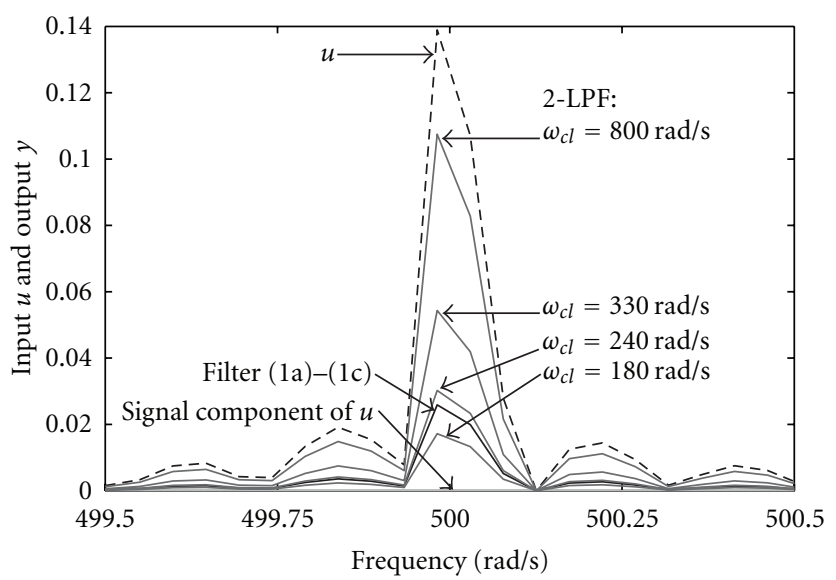

(c) Amplitude spectrum around $\omega=500 \mathrm{rad} / \mathrm{s}$

Figure 11: Comparison between the outputs of filter (1a)-(1c) with $F=9000 \mathrm{~s}^{-2}$ and $\alpha=3$ and 2-LPF with $\omega_{c l}=180,240,330$ and $800 \mathrm{rad} / \mathrm{s}$ for the input $u=\sin (100 t)+0.16 \sin (500 t)$. In (c), the amplitude of $u$ at $\omega=500 \mathrm{rad} / \mathrm{s}$ is not exactly 0.16 presumably because of the limited length of sampled data.

$F$ as $F>\Gamma^{-1}\left(G_{p}\right) \omega_{s}^{2} U_{s} / 2$. As a whole, one can see that, in order to achieve the requirements (a) and (b), the value of $F$ should satisfy the following relation:

$$
\frac{\Gamma^{-1}\left(G_{p}\right) \omega_{s}^{2} U_{s}}{2}<F<\frac{\omega_{n}^{2} U_{n p}}{2} .
$$

Thus, as a guideline for selecting $F$, in the case where the estimates of $\omega_{s}, U_{s}, \omega_{n}, U_{n p}$, and $G_{p}$ are known in advance, the value of $F$ should be simply set to satisfy (16). With such a value of $F$, filter (1a)-(1c) passes the signal component while attenuating the noise component down to an amplitude of $U_{n p}$. Note that if $\Gamma^{-1}\left(G_{p}\right) \omega_{s}^{2} U_{s} \geq \omega_{n}^{2} U_{n p}$, the guideline cannot be applied and the value of $U_{n p}$ or the value of $G_{p}$ must be reconsidered.

As a guideline for selecting $\alpha$, based on the discussion in Section 4, $\alpha$ should be set as $3 \leq \alpha \leq 4$. However, in applications where the transient time is not a major concern, it is advisable to set it as large as $4 \leq \alpha \leq 5$ because phase lag becomes smaller by making $\alpha$ larger, as indicated in Section 3.2.

\section{Numerical Example}

Filter (1a)-(1c) and the guidelines are now validated through numerical examples. The following signal is used as the input signal to filter (1a)-(1c):

$$
u=\sin (100 t)+0.16 \sin (500 t),
$$

where the first term and the second term on the right-hand side are here considered as the signal and noise components, respectively. Here, we assume that $u$ is a dimensionless quantity while $t$ is measured in $\mathrm{s}$. The requirements for filter (1a)-(1c) are set as follows:

(a) the amplitude of the noise component in the output should be smaller than $U_{n p}=0.075$, and

(b) the gain $G$ of the signal component should be $G_{p}=$ $-0.7 \mathrm{~dB}<G<0 \mathrm{~dB}$.

According to the guidelines, the value of $\alpha$ is set as $\alpha=3$. In addition, from Figure 10, one can know that $\Gamma^{-1}\left(G_{p}=\right.$ $-0.7) \approx 1.7$. Thus, the value of $F$ is set as $F=9000 \mathrm{~s}^{-2}$, which satisfies the relation $\Gamma^{-1}(-0.7) \times 100^{2} / 2<F<500^{2} \times 0.075 / 2$. 


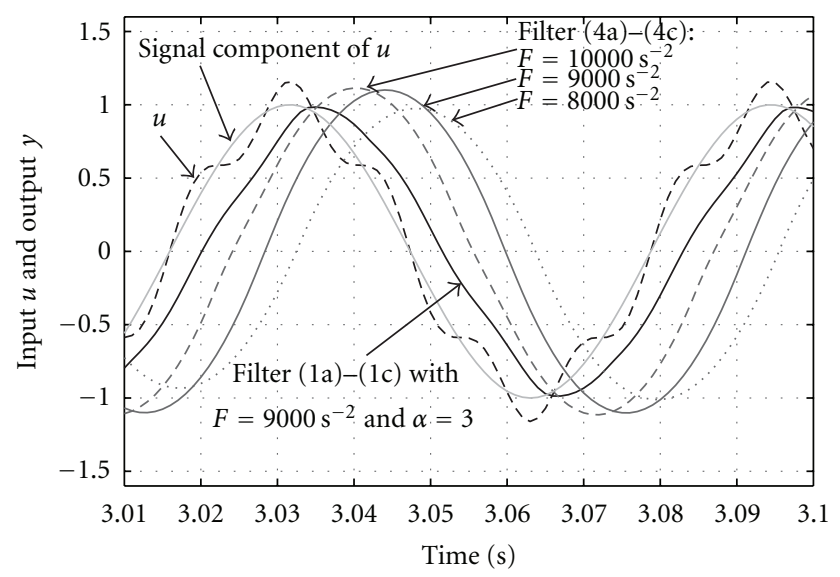

FIgure 12: Comparison between the outputs of filter (1a)-(1c) with $F=9000 \mathrm{~s}^{-2}$ and $\alpha=3$ and filter (4a)-(4c) with $F=8000,9000$, and $10000 \mathrm{~s}^{-2}$ for the input $u=\sin (100 t)+$ $0.16 \sin (500 t)$.

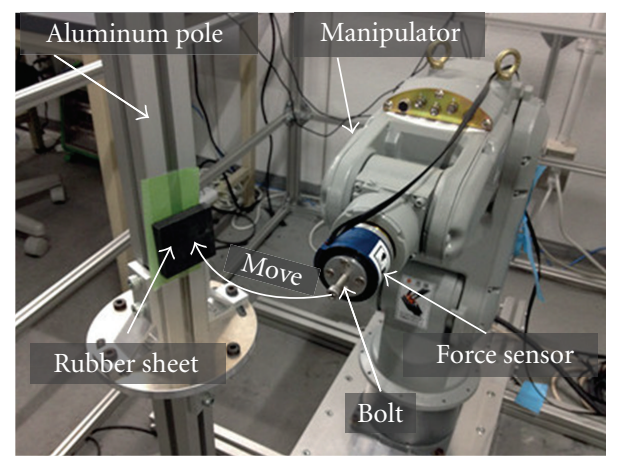

FIgURE 13: Experimental setup.

Figure 11 shows the comparison between the outputs of filter (1a)-(1c) and 2-LPF. Here, the input $u$ starts from $u=0$ at $t=0 \mathrm{~s}$, and the initial states of all filters are zeros at $t=$ $0 \mathrm{~s}$. Figure 11 only shows the data after $t=3.01 \mathrm{~s}$, where the outputs are in the steady state. In 2-LPF, cut-off frequencies $\omega_{c l}=180,240,330$, and $800 \mathrm{rad} / \mathrm{s}$ are used for comparison. In the rest part of this section, the sampling interval $T=$ $0.001 \mathrm{~s}$ is used for the numerical examples.

The figure shows that in filter (1a)-(1c), the output amplitude is 0.98 , which is equivalent to the gain $G=$ $-0.176 \mathrm{~dB}$. This satisfies the requirement (b), that is, $G>$ $G_{p}=-0.7 \mathrm{~dB}$. Besides that, the amplitude of the output at $\omega=500 \mathrm{rad} / \mathrm{s}$ is $U=0.026$, which satisfies the requirement (a), that is, $U<U_{n p}=0.075$. Thus, by using the guidelines, one can see that the two requirements are satisfied.

Figure 11 also shows the advantage of filter (1a)-(1c) over 2-LPF. Compared to filter (1a)-(1c), 2-LPF produces smaller phase lag only at $\omega_{c l}>330 \mathrm{rad} / \mathrm{s}$ and smaller noise amplitude only at $\omega_{c l}<240 \mathrm{rad} / \mathrm{s}$, as shown in Figures 11(b) and $11(\mathrm{c})$, respectively. Thus, one can see that 2-LPF cannot bring the output as close to the signal component in the input as filter (1a)-(1c) does by adjusting the cutoff frequency $\omega_{c l}$.
Filter (1a)-(1c) is also compared to filter $(4 a)-(4 c)$ in Figure 12. The figure shows the output of filter (1a)-(1c) with $F=9000 \mathrm{~s}^{-2}$ and $\alpha=3$ and those of filter $(4 \mathrm{a})-(4 \mathrm{c})$ with $F=8000,9000$, and $10000 \mathrm{~s}^{-2}$. It is shown that filter (4a)-(4c) produces larger phase lag than filter (1a)-(1c) does with the same $F$ value. This indicates the advantage of the use of $\alpha>1$. It is clear that, in the case of filter $(4 a)-(4 c)$, the phase lag can be reduced by using a larger $F$ value, but it sacrifices the noise removing capability, as also shown in Figure 12.

\section{Experiment}

This section reports a set of experimental results for validating filter (1a)-(1c) and the presented guidelines. Figure 13 shows the experimental setup, which consisted of a robot manipulator (MOTOMAN HP3-J, Yaskawa Electric Corporation) and an aluminum pole with a rubber sheet attached to it. The manipulator was equipped with a force sensor (Nitta Corporation) and a bolt installed on the force sensor. In the experiment, the manipulator was controlled so that the bolt head maintained contact with the rubber sheet with a particular contact force. Only the first joint (from the base) was controlled and the other joints were locked to fixed angles so that the end effector moves along the circular arc indicated in Figure 13. In the following descriptions, all quantities are measured in the translational system along the arc.

Admittance control [36-38] was used to control the contact force between the bolt head and the rubber sheet. Figure 14 shows the block diagram of the whole system. In admittance control, a virtual object having simple dynamics is considered in the controller, measured force signal is used to simulate the object's motion according to the force, and the manipulator is position controlled so as to follow the resultant object's motion. As long as the position control is accurate enough, the manipulator's response to external forces is close to that of the virtual object. It has been known that, however, such a control system can be unstable due to the phase lag resulted from many hardware factors (e.g., the latency in the controller and the compliance of the joint). The phase-lead compensator in Figure 14 was intended to attenuate this effect, but it also has a side effect of magnifying the noise. The filter in Figure 14 was for reducing the influence of the noise in the force sensor measurement. To maintain the stability, the filter should not produce much phase lag.

The expression of the admittance controller used in the experiment is given follows:

$$
\begin{aligned}
\ddot{q}= & \frac{-b \dot{q}-f_{f}-T_{f} \dot{f}_{f}+f_{d}}{m}, \\
\tau= & K_{P}(q-p)+K_{I} \int(q-p) d t \\
& +K_{D}(\dot{q}-\dot{p})+M \ddot{q} .
\end{aligned}
$$

Here, (18) represents the virtual object dynamics, where $f_{d}$ is the desired pressing force, $f_{f}$ is the filtered force, $T_{f}$ is a constant, and $q, m$, and $b$ are position, inertia, and viscosity 


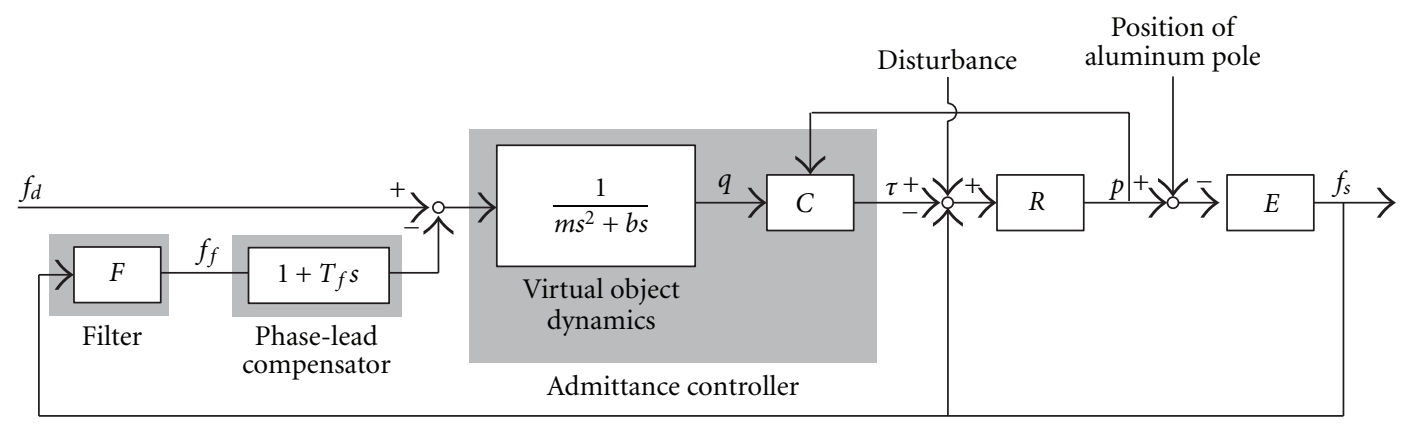

FIGURE 14: Block diagram of an admittance-controlled system ( $F$ : filter, $s$ : differentiator, $T_{f}$ : constant, $C$ : position controller, $R$ : manipulator dynamics, $E$ : environmental dynamics).

TABLE 1: Parameter values.

\begin{tabular}{lc}
\hline Parameter & Value \\
\hline$T$ & $0.001 \mathrm{~s}$ \\
$T_{f}$ & $0.0012 \mathrm{~s}$ \\
$f_{d}$ & $1 \mathrm{~N}$ \\
$m$ & $0.7 \mathrm{~kg}$ \\
$b$ & $6 \mathrm{~N} \cdot \mathrm{s} / \mathrm{m}$ \\
$M$ & $10.3 \mathrm{~kg}$ \\
$K_{P}$ & $5.17 \times 10^{4} \mathrm{~N} / \mathrm{m}$ \\
$K_{D}$ & $5.17 \times 10^{2} \mathrm{~N} \cdot \mathrm{s} / \mathrm{m}$ \\
$K_{I}$ & $1.03 \times 10^{5} \mathrm{~N} /(\mathrm{m} \cdot \mathrm{s})$ \\
\hline
\end{tabular}

of the object, respectively. In addition, (19) is a proportionalintegral-derivative position controller with inertia compensation, where $p$ is the position of the manipulator, $M$ is a constant, $\tau$ is the output force, and $K_{P}, K_{I}$, and $K_{D}$ are proportional, integral, and derivative gains, respectively.

In the measured force signal $f_{s}$, the maximum amplitude and frequency of the useful signal component were estimated as $15 \mathrm{~N}$ and $10 \mathrm{~Hz}(62.8 \mathrm{rad} / \mathrm{s})$, respectively, and the minimum frequency of the noise component was estimated as $200 \mathrm{~Hz}(1256.6 \mathrm{rad} / \mathrm{s})$. The requirements for filter (1a)-(1c) were set as follows:

(a) the amplitude of the noise component in the output should be smaller than $0.1 \mathrm{~N}$, and

(b) the gain $G$ of the useful signal component should be $G_{p}=-0.7 \mathrm{~dB}<G<0 \mathrm{~dB}$.

By applying the guidelines, the value of $\alpha$ was set as $\alpha=3$, and the value of $F$ was set as $F=65000 \mathrm{~N} / \mathrm{s}^{2}$, which satisfies the relation $\Gamma^{-1}(-0.7) \times(62.8)^{2} \times 15 / 2<F<(1256.6)^{2} \times$ $0.1 / 2$. The other parameters for the experiment were set as shown in Table 1.

The experimental results are shown in Figures 15 and 16. Figure 15 shows the data of the average magnitude of $\left|\dot{f}_{s}\right|$, which is defined as

$$
\mathrm{AMFD} \triangleq \int_{15}^{30} \frac{\left|\dot{f}_{s}(t)\right| d t}{(30-15)}
$$

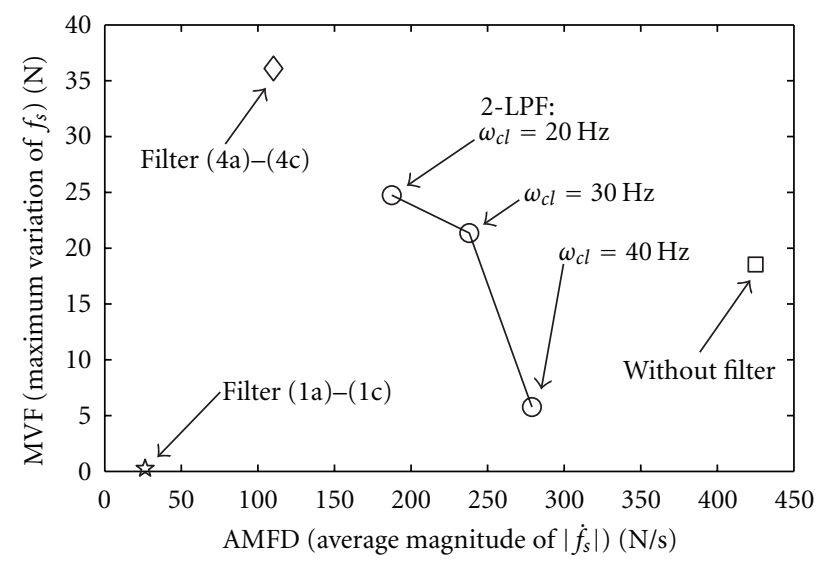

Figure 15: Comparison among filter (1a)-(1c) with $F=$ $65000 \mathrm{~N} / \mathrm{s}^{2}$ and $\alpha=3,2$-LPF with $\omega_{c l}=20,30$, and $40 \mathrm{~Hz}$, and filter (4a)-(4c) with $F=65000 \mathrm{~N} / \mathrm{s}^{2}$ and without filter.

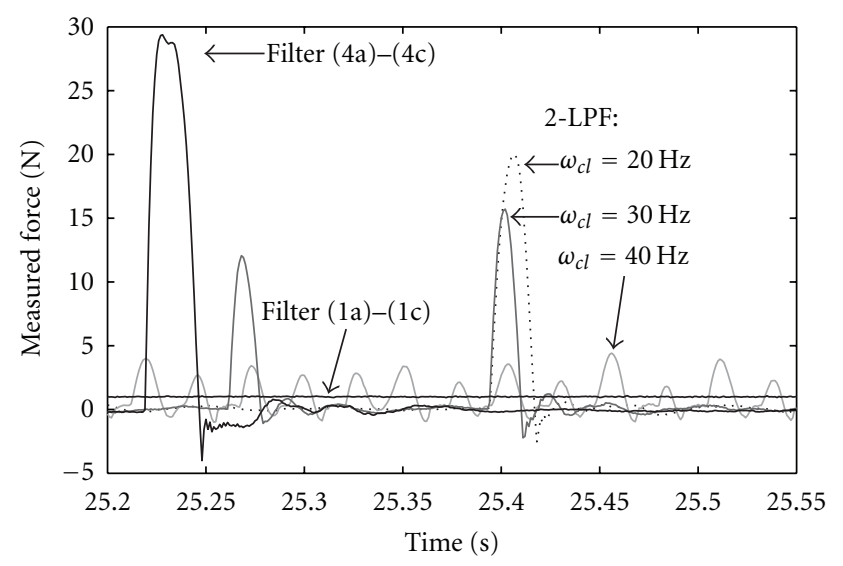

FIGURE 16: Typical contact force data obtained through filter (1a)(1c) with $F=65000 \mathrm{~N} / \mathrm{s}^{2}$ and $\alpha=3,2-\mathrm{LPF}$ with $\omega_{c l}=20,30$, and $40 \mathrm{~Hz}$, and filter (4a)-(4c) with $F=65000 \mathrm{~N} / \mathrm{s}^{2}$.

and the maximum variation of $f_{s}$, which is defined as

$$
\mathrm{MVF} \triangleq\left|\max _{15 \mathrm{~s} \leq t \leq 30 \mathrm{~s}} f_{s}(t)-\min _{15 s \leq t \leq 30 \mathrm{~s}} f_{s}(t)\right| .
$$

A large AMFD value indicates that there is a strong highfrequency vibration, and a large MVF value indicates that 
there is a strong low-frequency vibration. In addition, Figure 16 provides typical contact force data obtained through the three filters. Here, the experiment started from $t=0 \mathrm{~s}$, and the initial states of the three filters were zeros at $t=0 \mathrm{~s}$. Figures 15 and 16 only use the data after $t=15 \mathrm{~s}$, that is, time period after the contact had occurred. In 2-LPF, cut-off frequency $\omega_{c l}=20,30$, and $40 \mathrm{~Hz}$, and in filter (4a)(4c), $F=65000 \mathrm{~N} / \mathrm{s}^{2}$ were used.

The two figures show that filter (1a)-(1c) reduced both AMFD and MVF compared to the other filters, and the manipulator successfully maintained a contact force of $1 \mathrm{~N}$, which is almost equal to $f_{d}$. The two figures also show that, in the case of 2-LPF, AMFD and MVF cannot be simultaneously reduced as close to the values as filter (1a)-(1c) did by adjusting the cut-off frequency $\omega_{c l}$. In the case of filter (4a)(4c), one can observe that MVF was the largest among the three filters. These results clearly show the advantage of filter (1a)-(1c) over 2-LPF and filter (4a)-(4c).

\section{Conclusions}

This paper has quantitatively evaluated the performance of filter (1a)-(1c), which is a parabolic sliding mode filter proposed in [30], based on the frequency and time domain characteristics. Based on the evaluation results, simple guidelines for selecting two parameter, $F$ and $\alpha$, of the filter have been provided. In addition, filter (1a)-(1c) and the presented guidelines have been validated through numerical examples and experiments. The evaluation results show that filter (1a)-(1c) has better frequency and time responses than those of the second-order Butterworth low-pass filter (2LPF) and filter (4a)-(4c), which is a conventional parabolic sliding mode filter proposed in [17-19]. Specifically, in the frequency domain, the noise removing capability of filter (1a)-(1c) is almost the same as that of 2-LPF, but its phase lag is smaller (maximum 150 degree) than that of 2-LPF (maximum 180 degree). In addition, the phase lag caused by filter (1a)-(1c) is smaller than that of filter (4a)(4c) with appropriate selection of the parameters. In the time domain, filter (1a)-(1c) produces smaller overshoot than 2-LPF and filter (4a)-(4c), while maintaining short transient time, by using appropriately selected $\alpha$ value. The guidelines state that $F$ should be set to satisfy (16), which depends on some estimated characteristics of the input and some desired characteristics of the output. Moreover, the guidelines recommend that $\alpha$ should be set as $3 \leq$ $\alpha \leq 4$. The presented numerical examples and experimental results confirm the effectiveness of filter (1a)-(1c) and the parameter selection guidelines.

One limitation of this paper is that the performance of filter (1a)-(1c) is only evaluated through numerical methods. Thus, theoretical validation of the guidelines should be addressed in future study.

\section{Acknowledgments}

This work was supported by Grant-in-Aid for Scientific Research B (no. 24360098) from Japan Society for the Promotion of Science (JSPS). The authors are grateful to
Mr. Tomohiro Kumon for his technical support in the experiments.

\section{References}

[1] N. C. Gallagher and L. W. Gary, "A theoretical analysis of the properties of median filters," IEEE Transactions on Acoustics, Speech, and Signal Processing, vol. 29, no. 6, pp. 1136-1141, 1981.

[2] V. G. Moshnyaga and K. Hashimoto, "An efficient implementation of 1-D median filter," in Proceedings of the 52nd IEEE International Midwest Symposium on Circuits and Systems (MWSCAS '09), pp. 451-454, August 2009.

[3] F. Janabi-Sharifi, V. Hayward, and C. S. J. Chen, "Discrete-time adaptive windowing for velocity estimation," IEEE Transactions on Control Systems Technology, vol. 8, no. 6, pp. 10031009, 2000.

[4] G. Welch and G. Bishop, "An introduction to the Kalman filter," Tech. Rep. TR 95-041, Department of Computer Science, University of North Carolina, 1995.

[5] P. Hargrave, "A tutorial introduction to Kalman filtering," in Proceedings of the IEE Colloquium on Kalman Filters: Introduction, Applications and Future Developments, pp. 1-6, 1989.

[6] K. K. Ahn and D. Q. Truong, "Online tuning fuzzy PID controller using robust extended Kalman filter," Journal of Process Control, vol. 19, no. 6, pp. 1011-1023, 2009.

[7] C. A. Lightcap and S. A. Banks, "An extended kalman filter for real-time estimation and control of a rigid-link flexiblejoint manipulator," IEEE Transactions on Control Systems Technology, vol. 18, no. 1, pp. 91-103, 2010.

[8] J. J. E. Slotine, J. K. Hedrick, and E. A. Misawa, "On sliding observers for nonlinear systems," Journal of Dynamic Systems, Measurement, and Control, vol. 109, no. 3, pp. 245-252, 1987.

[9] F. Chen and M. W. Dunnigan, "Comparative study of a sliding-mode observer and Kalman filters for full state estimation in an induction machine," IEE Proceedings on Electric Power Applications, vol. 149, no. 1, pp. 53-64, 2002.

[10] A. Alessandri, "Sliding-mode estimators for a class of nonlinear systems affected by bounded disturbances," International Journal of Control, vol. 76, no. 3, pp. 226-236, 2003.

[11] A. Levant, "Robust exact differentiation via sliding mode technique," Automatica, vol. 34, no. 3, pp. 379-384, 1998.

[12] J. A. Moreno and M. Osorio, "A Lyapunov approach to secondorder sliding mode controllers and observers," in Proceedings of the 47th IEEE Conference on Decision and Control (CDC '08), pp. 2856-2861, December 2008.

[13] N. K. M’sirdi, A. Rabhi, L. Fridman, J. Davila, and Y. Delanne, "Second order sliding mode observer for estimation of velocities, wheel sleep, radius and stiffness," in Proceedings of the 2006 American Control Conference, pp. 3316-3321, June 2006.

[14] J. Davila, L. Fridman, and A. Levant, "Second-order slidingmode observer for mechanical systems," IEEE Transactions on Automatic Control, vol. 50, no. 11, pp. 1785-1789, 2005.

[15] S. Solvar, V. Le, M. Ghanes, J. P. Barbot, and G. Santomenna, "Sensorless second order sliding mode observer for induction motor," in Proceedings of the IEEE International Conference on Control Applications (CCA '10), pp. 1933-1938, September 2010.

[16] N. K. M'Sirdi, A. Rabhi, L. Fridman, J. Davila, and Y. Delanne, "Second order sliding-mode observer for estimation of vehicle dynamic parameters," International Journal of Vehicle Design, vol. 48, no. 3-4, pp. 190-207, 2008. 
[17] J. Q. Han and W. Wang, "Nonlinear trackingdifferentiator," Journal of Systems Science and Mathematical Science, vol. 14, no. 2, pp. 177-183, 1994 (Chinese).

[18] T. Emaru and T. Tsuchiya, "Research on estimating the smoothed value and the differential of the sensor inputs by using sliding mode system (application to ultrasonic wave sensor)," Transactions of the Japan Society of Mechanical Engineers C, vol. 66, no. 652, pp. 3947-3954, 2000 (Japanese).

[19] T. Emaru and T. Tsuchiya, "Research on estimating smoothed value and differential value by using sliding mode system," IEEE Transactions on Robotics and Automation, vol. 19, no. 3, pp. 391-402, 2003.

[20] J. Q. Han, W. Wang, T. Emaru, and T. Tsuchiya, "Comments on 'research on estimating smoothed value and differential value by using sliding mode system,', IEEE Transactions on Robotics, vol. 21, no. 6, p. 1241, 2005.

[21] T. Emaru and T. Tsuchiya, "Research on signal tracking performance of nonlinear digital filter ESDS," Transactions of the Japan Society of Mechanical Engineers C, vol. 70, no. 8, pp. 2352-2359, 2004 (Japanese).

[22] J. Q. Han and Y. C. Huang, "Frequency characteristic of second-order tracking-differentiator," Mathematics in Practice and Theory, vol. 33, no. 3, pp. 71-74, 2003 (Chinese).

[23] B. Z. Guo and Z. L. Zhao, "On convergence of tracking differentiator," International Journal of Control, vol. 84, no. 4, pp. 693-701, 2011.

[24] Y. X. Su, B. Y. Duan, C. H. Zheng, Y. F. Zhang, G. D. Chen, and J. W. Mi, "Disturbance-rejection high-precision motion control of a stewart platform," IEEE Transactions on Control Systems Technology, vol. 12, no. 3, pp. 364-374, 2004.

[25] Z. Gao, Y. Huang, and J. Han, "An alternative paradigm for control system design," in Proceedings of the 40th IEEE Conference on Decision and Control (CDC '01), pp. 4578-4585, December 2001.

[26] J. Han, "From PID to active disturbance rejection control," IEEE Transactions on Industrial Electronics, vol. 56, no. 3, pp. 900-906, 2009.

[27] Y. X. Su, D. Sun, and B. Y. Duan, "Design of an enhanced nonlinear PID controller," Mechatronics, vol. 15, no. 8, pp. 10051024, 2005.

[28] Y. X. Su, B. Y. Duan, and C. H. Zheng, "Nonlinear PID control of a six-DOF parallel manipulator," IEE Proceedings on Control Theory and Applications, vol. 151, no. 1, pp. 95-102, 2004.

[29] X. Peng, S. Cheng, and J. Wen, "Application of nonlinear PID controller in superconducting magnetic energy storage," International Journal of Control, Automation and Systems, vol. 3, no. 2, pp. 296-301, 2005.

[30] S. Jin, R. Kikuuwe, and M. Yamamoto, "Real-time quadratic sliding mode filter for removing noise," Advanced Robotics, vol. 26, no. 8-9, pp. 877-896, 2012.

[31] S. Jin, R. Kikuuwe, and M. Yamamoto, "Improved velocity feedback for position control by using a quadratic sliding mode filter," in Proceedings of the 11th International Conference on Control, Automation and Systems, pp. 1207-1212, 2011.

[32] R. Kikuuwe, N. Takesue, and H. Fujimoto, "A control framework to generate nonenergy-storing virtual fixtures: use of simulated plasticity," IEEE Transactions on Robotics, vol. 24, no. 4, pp. 781-793, 2008.

[33] R. Kikuuwe, S. Yasukouchi, H. Fujimoto, and M. Yamamoto, "Proxy-based sliding mode control: a safer extension of PID position control," IEEE Transactions on Robotics, vol. 26, no. 4, pp. 670-683, 2010.
[34] V. Acary and B. Brogliato, "Implicit Euler numerical scheme and chattering-free implementation of sliding mode systems," Systems and Control Letters, vol. 59, no. 5, pp. 284-293, 2010.

[35] H. K. Khalil, Nonlinear Systems, Prentice Hall, New York, NY, USA, 3rd edition, 2002.

[36] A. C. Schouten, E. de Vlugt, J. J. B. van Hilten, and F. C. T. van der Helm, "Design of a torque-controlled manipulator to analyse the admittance of the wrist joint," Journal of Neuroscience Methods, vol. 154, no. 1-2, pp. 134-141, 2006.

[37] C. Ott, R. Mukherjee, and Y. Nakamura, "Unified impedance and admittance control," in Proceedings of the IEEE International Conference on Robotics and Automation (ICRA '10), pp. 554-561, May 2010.

[38] R. Q. van der Linde and P. Lammertse, "HapticMaster-a generic force controlled robot for human interaction," Industrial Robot, vol. 30, no. 6, pp. 515-524, 2003. 

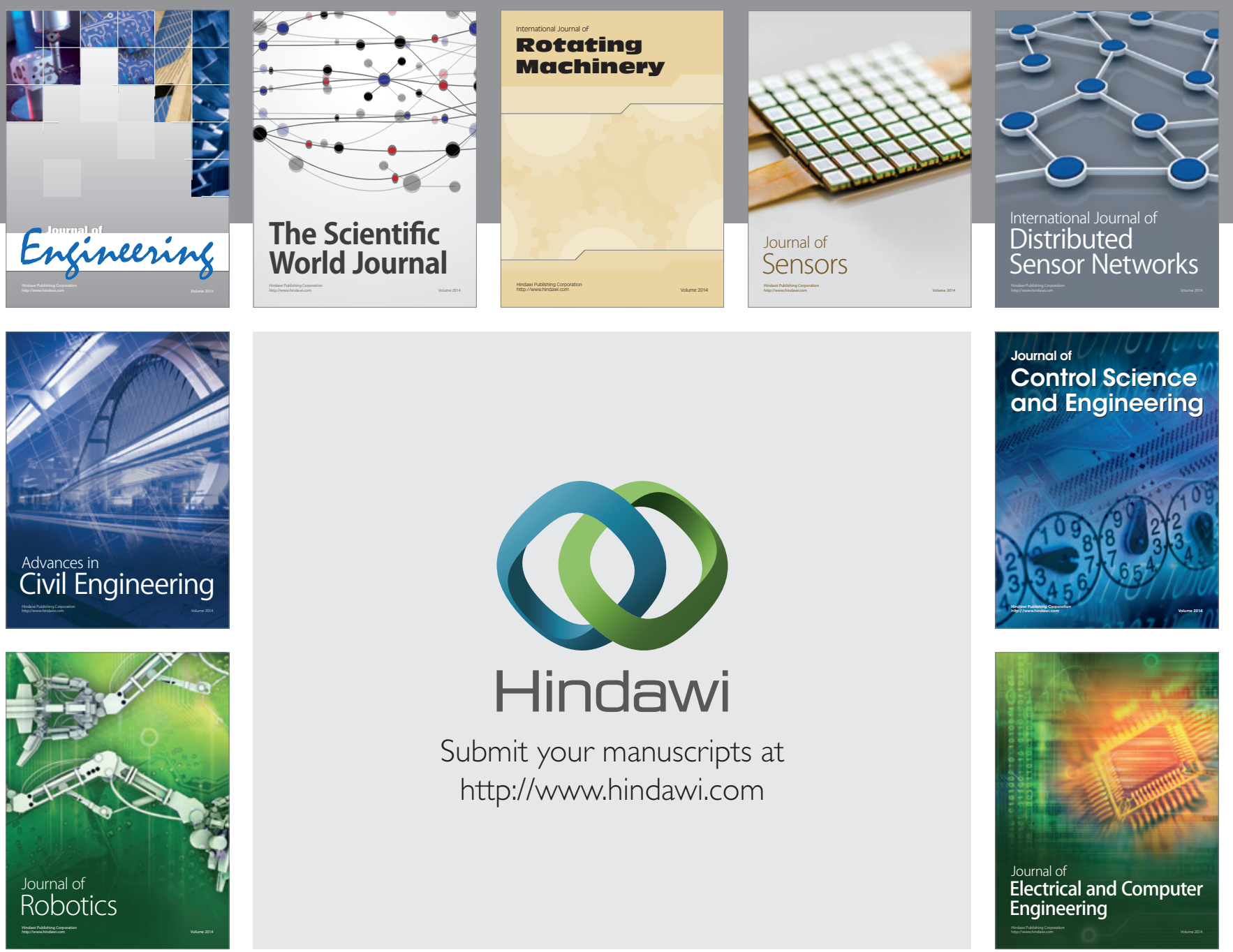

Submit your manuscripts at

http://www.hindawi.com
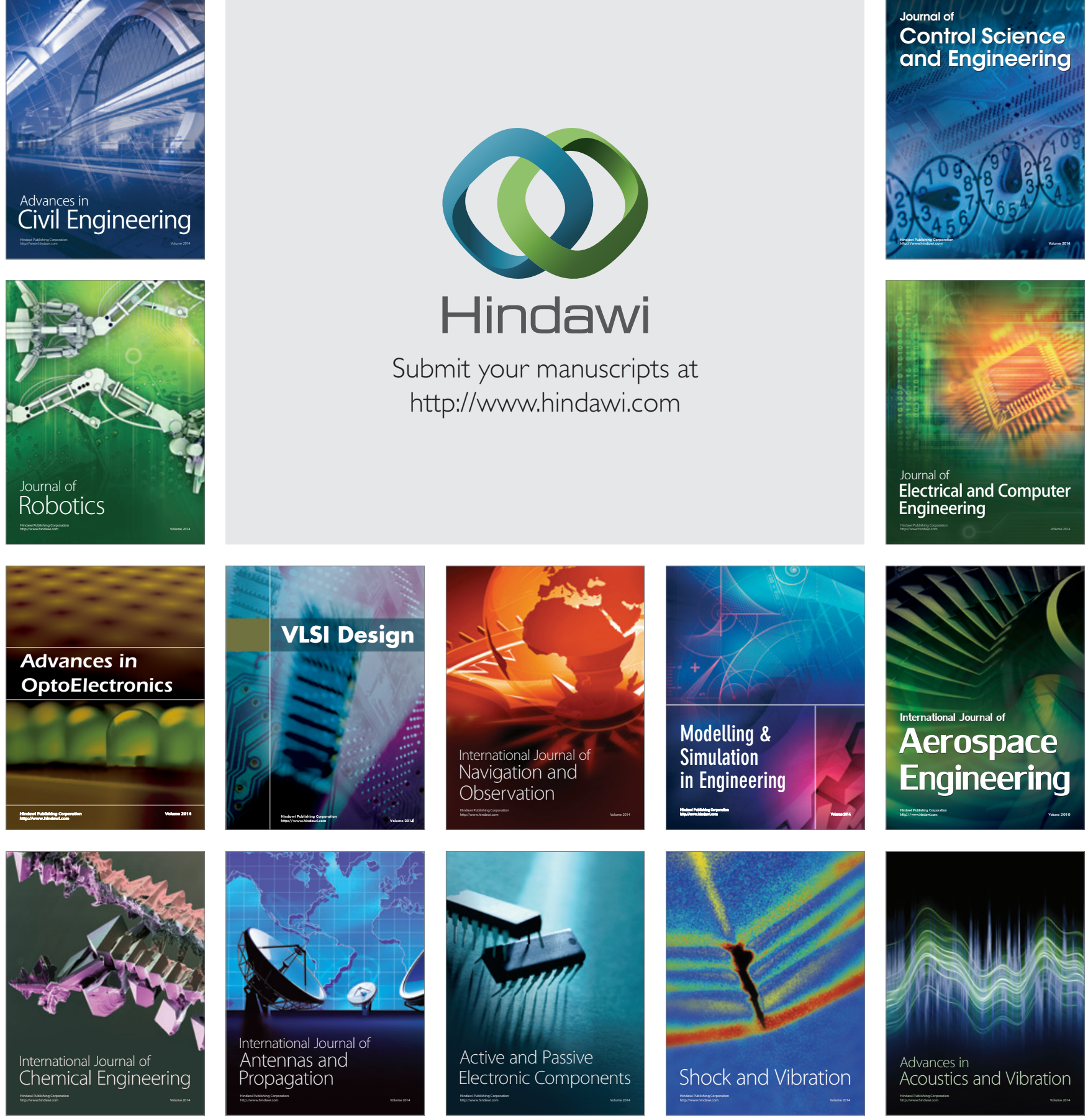